\title{
Analysis of Liquid Structure without Construction of Any Structure Models by the X-Ray Scattering Method
}

\author{
Misaki Katayama, Shingo Ashiki, and Kazuhiko Ozutsumi ${ }^{\dagger}$ \\ Department of Applied Chemistry, Faculty of Science and Engineering, Ritsumeikan University, \\ 1-1-1 Noji-Higashi, Kusatsu 525-8577, Japan
}

\begin{abstract}
A simple approach for determining a liquid structure using X-ray scattering data, in which a liquid structure is uniquely evaluated without construction of any plausible structure models, has been applied to liquid acetonitrile, acetone and cyclohexane. For a pair of molecules, a given point within a molecule is located at the origin with a given molecular orientation. The site of the given point of another molecule is defined by the polar coordinates and the molecular orientation is treated by three Eulerian angles. These parameters are optimized by a non-linear least-squares calculation applied to $\mathrm{X}$-ray scattering data. The reliability of the method was examined by determining the liquid structure of polar acetonitrile and the obtained intermolecular interatomic distances are in good agreement with the previously reported values. The method was then successfully applied to the determination of the liquid structure of acetone and cyclohexane. Especially for nonpolar cyclohexane, the construction of a variety of plausible structural models is very difficult. It was revealed that acetone has an ordered liquid arrangement similar to that found in its crystal, although the intermolecular distances in liquid acetone are different from those in the crystal. On the other hand, the liquid structure of cyclohexane is disordered.
\end{abstract}

(Received May 8, 2007; Accepted June 4, 2007; Published August 10, 2007)

\section{Introduction}

Since a study on liquid paraffin by Debye and Scherrer, ${ }^{1}$ a large number of structural studies on various liquids have been conducted by the X-ray scattering method. The structure of liquid water in various conditions has been examined by many investigators. ${ }^{2,3}$ Structural information on organic liquids has also been accumulated. ${ }^{47}$

Due to large fluctuations caused by molecular motions in long distant regions, analyses of a liquid structure by the X-ray scattering method require one to make up a local structure model, i.e., an aggregate consisting of several liquid molecules, which best reproduces the X-ray scattering data.

The simplest approach to deduce a structure model of a liquid will refer to a partial structure present in its crystal when the crystal structure has been determined. The interstitial model of liquid water by Narten $^{8}$ is the most famous example. Determinations of the local structure of organic liquids such as benzene, formamide, dimethyl sulfoxide, and $\mathrm{N}$-methylacetamide at ambient temperature (their melting points are 5.493, 2.55, 18.55 , and $30.55^{\circ} \mathrm{C}$, respectively) are successful examples based on the partial structure in the crystal. ${ }^{9-12}$ In order to obtain further information on the longer range intermolecular interactions of molecular liquids, Iijima and Nishikawa proposed the reciprocal space expansion in the analysis of X-ray scattering data. ${ }^{13,14}$ However, these procedures do not apply if the temperature of a liquid is far from its melting point because of large differences of densities between solids and liquids. The intermolecular distances among molecules in a liquid are quite different from those in a solid phase.

$\dagger$ To whom correspondence should be addressed.

E-mail: ozutsumi@se.ritsumei.ac.jp
Another approach to construct a liquid structure is one that uses physicochemical properties of liquid molecules. When a liquid is protic and has a hydrogen-bonding ability, a two- or three-dimensional hydrogen-bonded network should be taken into account. For an aprotic polar liquid molecule, a negatively charged atom of a molecule approaches a positively charged atom of another molecule according to the charge distributions of the molecules. Also, molecules are piled up interacting with their dipole moments in an antiparallel way. Considering these patterns, one searches for molecular arrangements which reproduce X-ray scattering data well by a trial-and-error method. A good example is the structural determination of liquid acetonitrile. ${ }^{15-19}$ However, analysis by this approach is a laborious task. Thus, a simple approach for determining a liquid structure without constructing any plausible structure models is necessary.

Two methods have been used frequently for studying liquid structures without constructing any plausible models, i.e., the reverse Monte Carlo (RMC) ${ }^{20}$ method and the empirical potential structure refinement (EPSR $)^{21}$ method. The RMC method is the computer simulation based on the Metropolis Monte Carlo procedure: a particle or a molecule moves so as to decrease the difference between the experimental scattering data and calculated ones. The EPSR method is a Monte Carlo simulation and involves the optimization process of the potentials. However, these computer simulations are time-consuming and it is hard to use these methods for a large number of structural investigations of liquid mixtures at various concentration ratios. Again, it is obvious that a simple approach for determining a liquid structure without construction of any plausible structure models is needed; such an approach could easily be extended to investigations of liquid mixtures.

For an $n$-molecule system, the position and orientation of the $(n-1)$ molecules around the central molecule are defined by the polar coordinates and Eulerian angles. It is possible to optimize 
these polar coordinates and Eulerian angles by fitting to X-ray scattering data. This approach was previously reported by Bertagnolli et al. and was adopted for the determination of the liquid structure of acetone using X-ray and neutron scattering data. ${ }^{22}$ Their result was that an aggregate consisting of 6 acetone molecules well reproduced both X-ray and neutron data. However, in our experience 3 or 4 molecules are usually enough to interpret X-ray scattering data. This suggests that some errors may have been involved in their fitting procedure. Thus, it is important to establish this approach as a versatile method in wider use and to refine the liquid structure of acetone.

In the present report, we first describe the principle of the simple approach and its application to the determination of a local structure of liquid consisting of several liquid molecules. Next, by examining the liquid structure of acetonitrile, we checked the applicability of the present method, because the antiparallel zigzag molecular arrangement in liquid acetonitrile has been widely accepted by many researchers. ${ }^{15-19}$ The reliability of the method was confirmed by the fact that obtained structural parameters for the intermolecular interactions were in good agreement with the literature values. Then, we measured and analyzed the X-ray scattering data of liquid acetone in order to refine the result published by Bertagnolli et al. ${ }^{22}$ Finally, the liquid structure of cyclohexane was studied. The liquid molecule is nonpolar and a construction of a liquid structure model is not easy. In fact, X-ray and neutron scattering measurements were carried out so far,,$^{4,5,23,24}$ but no detailed intermolecular arrangement has never been given yet.

\section{Experimental}

Acetonitrile, acetone and cyclohexane of reagent grade (Wako, Japan) were used. Acetonitrile was distilled under atmospheric pressure after pretreatment with molecular sieve $3 \mathrm{~A}$ for several weeks to remove water. The resulting distillate was kept in a dark bottle over molecular sieve $3 \mathrm{~A}$ to avoid water. Acetone and cyclohexane were purified by distillation under atmospheric pressure.

X-ray scattering measurements were carried out on a ROTAFLEX RU-300 (Rigaku, Japan), which was specially designed for the charge coupled device (CCD) detector (Quantum 1, ADSC). Details of the equipment were described elsewhere. ${ }^{19}$ Data were collected with the transmission geometry; an Mo rotating anode generator was operated at 50 $\mathrm{kV}$ and $240 \mathrm{~mA}$. A graphite (002) monochromator was used to obtain the Mo $K_{\alpha}$ line $(\lambda=71.07 \mathrm{pm})$ by eliminating the Mo $K_{\beta}$ emission. The resulting monochromatized beam was collimated to a diameter of $1.0 \mathrm{~mm}$ before irradiation to a sample. A liquid was shielded in a glass tube with the inner diameter of $2.0 \mathrm{~mm}$ and the wall thickness of $0.01 \mathrm{~mm}$. X-rays scattered by a liquid were detected by the CCD detector, which was cooled at $223 \mathrm{~K}$ and was stepwise moved at scattering angles $2 \theta=0,40,80$ and $120^{\circ}$ to cover the whole angle range of $140^{\circ}$, which corresponds to the scattering vector $s\left(=4 \pi \lambda^{-1} \sin \theta\right)$ range from 0 to $16.6 \times 10^{-2} \mathrm{pm}^{-1}$.

\section{Treatments of X-Ray Scattering Data}

Recorded intensities on the CCD detector as two-dimensional signals were corrected for the background. Then, the twodimensional data were integrated to give one-dimensional data, details of which were described elsewhere. ${ }^{19}$ The resulting onedimensional data were corrected for cell scattering, polarization, ${ }^{25}$ absorption, and multiple scattering ${ }^{26}$ by usual ways.

The structure function $i(s)$ was derived from the corrected intensities $I_{\text {corr }}(s)$ according to the following equation:

$$
i(s)=K I_{\text {corr }}(s)-\operatorname{del}(s) \sum_{\mathrm{i}} n_{\mathrm{i}} I_{\mathrm{i}}^{\mathrm{inc}}(s)-\sum_{\mathrm{i}}\left\{\left[f_{\mathrm{i}}(s)+\Delta f_{\mathrm{i}}^{\prime}\right]^{2}+\left(\Delta f_{\mathrm{i}}^{\prime \prime}\right)^{2}\right\}
$$

where $K$ denotes the normalization factor, which can be obtained by the high-angle region method or the Krough-Moe-Norman method, ${ }^{27,28} n_{\mathrm{i}}$ is the number of atom $\mathrm{i}$ in a unit volume involving one liquid molecule, and $I_{\mathrm{i}}^{\mathrm{inc}}(s)$ is the incoherent scattering intensity of atom . $^{29}$ A function representing the portion of incoherent radiation reaching the detector is $\operatorname{del}(s)$, and for the present transmission geometry $\operatorname{del}(s)=1$. The atomic scattering factor of atom $\mathrm{i}$ is denoted by $f_{\mathrm{i}}(s)$ and the real and imaginary parts of anomalous dispersion by $\Delta f_{i}^{\prime}$ and $\Delta f_{i}^{\prime \prime}$, respectively. ${ }^{30}$

The radial distribution function $D(r)$ was calculated by the Fourier transformation of the structure function $i(s)$ :

$$
D(r)=4 \pi r^{2} \rho_{0}+(2 r / \pi) \int_{0}^{S_{\max }} \operatorname{si}(s) M(s) \sin (r s) \mathrm{d} s
$$

where $\rho_{0}$ denotes the average electron density in the unit volume of the system. $M(s)$ is a modification function. The structure function and radial distribution function were calculated by using the program KURVLR. ${ }^{31}$

The theoretical structure function $i_{\text {calc }}(s)$ is obtained from the structure parameters, i.e., the distance between atoms $\mathrm{i}$ and $\mathrm{j}, r_{\mathrm{ij}}$, the temperature factor of the $\mathrm{i}-\mathrm{j}$ atom pair, $b_{\mathrm{ij}}$, and the number of the $\mathrm{i}-\mathrm{j}$ atom pair, $n_{\mathrm{ij}}$, by the following equation:

$$
\begin{aligned}
i_{\text {calc }}(s)=\sum_{\mathrm{i}} \sum_{\mathrm{j}} n_{\mathrm{ijj}}\left[\left\{f_{\mathrm{i}}(s)+\Delta f_{\mathrm{i}}^{\prime}\right\}\left\{f_{\mathrm{j}}(s)+\Delta f_{\mathrm{j}}^{\prime}\right\}+\Delta f_{\mathrm{i}}^{\prime \prime} \cdot \Delta f_{\mathrm{j}}^{\prime \prime}\right] \times \\
\left\{\sin \left(s r_{\mathrm{ij}}\right) /\left(s r_{\mathrm{ij}}\right)\right\} \exp \left(-b_{\mathrm{ij}} s^{2}\right)
\end{aligned}
$$

The temperature factor $b_{\mathrm{ij}}$ is related to the mean square displacement $\Delta r_{\mathrm{ij}}{ }^{2}$ of the $\mathrm{i}-\mathrm{j}$ atom pair in the form $b_{\mathrm{ij}}=\Delta r_{\mathrm{ij}}^{2} / 2$. The theoretical function $i_{\text {calc }}(s)$ is the sum of intramolecular and intermolecular parts as follows:

$$
i_{\text {calc }}(s)=i_{\text {intra }}(s)+i_{\text {inter }}(s)
$$

\section{Principle for the Determination of Molecular Orientation}

For a pair of molecules I and II, a given point within a molecule I is located at the origin with a given molecular orientation. The site of the given point of another molecule II is defined by the polar coordinates, $R, \Theta, \Phi$, and the molecular orientation is treated by three Eulerian angles $\psi, \theta, \phi$. We then call these the intermolecular structure parameters. The parameters are optimized by a non-linear least-squares calculation applied to X-ray scattering data. Each atom-atom distance, $r_{\mathrm{ij}}$, between the molecules I and II is obtained from the following equation:

$$
r_{\mathrm{ij}}=\sqrt{\left(x_{\mathrm{i}}-x_{\mathrm{j}}\right)^{2}+\left(y_{\mathrm{i}}-y_{\mathrm{j}}\right)^{2}+\left(z_{\mathrm{i}}-z_{\mathrm{j}}\right)^{2}}
$$

where $x_{\mathrm{i}}, y_{\mathrm{i}}$, and $z_{\mathrm{i}}$ are Cartesian coordinates of an atom $\mathrm{i}$ within the molecule I situated at the origin, and $x_{\mathrm{j}}, y_{\mathrm{j}}$, and $z_{\mathrm{j}}$ are those of an atom $\mathrm{j}$ within another molecule II. The latter coordinates are related with the former ones by the intermolecular structure parameters as follows:

$$
\begin{aligned}
x_{\mathrm{j}}=(\cos \phi \cos \theta \cos \psi-\sin \phi \sin \psi) \cdot x_{\mathrm{i}}+ \\
\\
(-\sin \phi \cos \theta \cos \psi-\cos \phi \sin \psi) \cdot y_{\mathrm{i}}+ \\
(\sin \theta \cos \psi) \cdot z_{\mathrm{i}}+R \cos \Phi \cos \Theta
\end{aligned}
$$



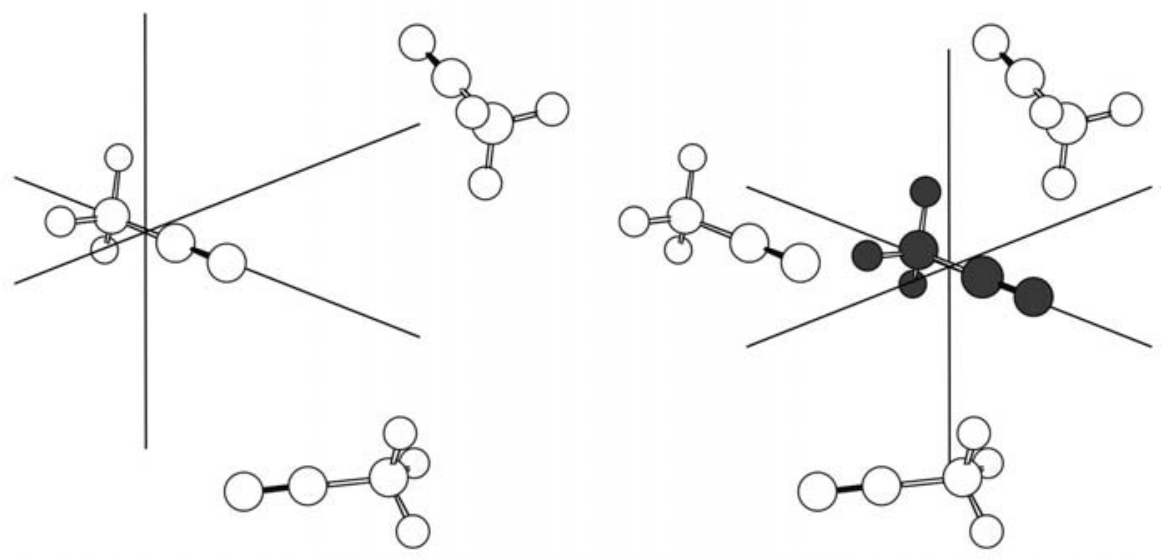

Fig. 1 Two types of initial distribution of molecules. Shaded atoms on the right hand side are those with no atomic scattering power.

$$
\begin{aligned}
& y_{\mathrm{j}}=(\cos \phi \cos \theta \cos \psi+\sin \phi \sin \psi) \cdot x_{\mathrm{i}}+ \\
&(-\sin \phi \cos \theta \sin \psi+\cos \phi \cos \psi) \cdot y_{\mathrm{i}}+ \\
&(\sin \theta \sin \psi) \cdot z_{\mathrm{i}}+R \cos \Phi \sin \Theta \\
& z_{\mathrm{j}}=-\cos \phi \sin \theta \cdot x_{\mathrm{i}}+\sin \phi \sin \theta \cdot y_{\mathrm{i}}+\cos \theta \cdot z_{\mathrm{i}}+R \sin \Phi
\end{aligned}
$$

where the first, second, and third terms come from the rotational operations and the fourth one comes from the translation. Moreover, we introduce the intermolecular temperature factor $B$ to obtain the temperature factor $b_{\mathrm{ij}}$ according to the following equation:

$$
b_{\mathrm{ij}}=\left(r_{\mathrm{ij}} \times B\right)^{2} / 2
$$

In the present study, the $B$ value was set to 0.1 because a value around 0.1 was usually accepted in the references. ${ }^{10,11,19}$ The theoretical reduced intensities for intermolecular interactions, $i_{\text {inter }}(s)$, are obtained from Eq. (3) and intermolecular structure parameters are refined by the least-squares method to minimize $u$ :

$$
u=\sum_{S_{\min }}^{S_{\max }} s^{2}\left\{i_{\text {inter }}(s)-\left[i_{\text {obsd }}(s)-i_{\text {intra }}(s)\right]\right\}^{2}
$$

where $s_{\min }$ and $s_{\max }$ are the lower and upper limits of the $s$ value selected. Calculations were carried out by our non-linear leastsquares program based on an algorithm proposed by Marquardt. ${ }^{32}$

\section{Practical Procedure for Determining Liquid Structure}

In order to determine the liquid structure, one needs several liquid molecules that are distributed randomly in a space before least-squares refinements. Two methods are possible for the distribution of molecules; these are shown in Fig. 1 for the 3molecule system of acetonitrile as an example.

The first method is depicted on the left hand side of Fig. 1. For an $n$-molecule system, one molecule is located at the origin and $(n-1)$ molecules surround the central molecule. In the course of the least-squares treatments, $6(n-1)$ parameters are optimized, except for the intermolecular temperature factors, by calculating interatomic distances between ${ }_{n} C_{2}$ pairs of the molecules. However, since one molecule is fixed at the origin, this method is limited to neat liquids. Moreover, even in a treatment of a neat liquid, this method is never applicable if several conformers are present in the liquid in comparable concentrations.

The second method is based on the distribution of $(n+1)$ molecules. Initially, one molecule at the origin is randomly surrounded by $n$ molecules. After the distribution, the Cartesian coordinates of the central molecule remain but the molecule disappears. This means that the atoms in the molecule at the origin have no X-ray scattering power. During the least-squares calculations, the sites and orientations of $n$ surrounding molecules relative to the central molecule, i.e., $6 n$ parameters, are refined by calculating interatomic distances between ${ }_{n} C_{2}$ pairs of the molecules. The atoms that have no X-ray scattering power are shaded in Fig. 1 (right hand side). Because different kinds of molecules can be simultaneously situated at the origin, the different kinds of molecules are distributed in a space.

In both cases the number $n$ was determined as follows. At first two molecules were situated in a space and a least-squares calculation was carried out. If the fit between the measured $\mathrm{X}$ ray scattering data and calculated values is not good, one additional molecule was then introduced in a space and a leastsquares calculation was again performed. This procedure was repeated until the experimental X-ray scattering data were well reproduced by the obtained intermolecular interaction parameters and introduction of one more molecule did not improve the result. When we analyzed the liquid structures of acetonitrile and cyclohexane, the two methods gave virtually the same results. Furthermore, several initial distributions of molecules were examined by randomly changing both sites and orientations of molecules, but the obtained results were not affected by the initial configurations. As stated above, the latter method is preferable in an extension of the present procedure for the intermolecular structural determination to liquid mixtures, although the number of parameters to be optimized is smaller in the former case than in the latter.

\section{Liquid Acetonitrile}

The structure function $i(s)$ multiplied by $s$ for acetonitrile is shown in Fig. 2. The oscillation (dotted line) in the high $s$ region of $6<s / 10^{-2} \mathrm{pm}^{-1}<16$ on the $\operatorname{si}(s)$ curve is due to the intramolecular interactions of an acetonitrile molecule. The differential radial distribution function $\left(D(r)-4 \pi r^{2} \rho_{0}\right)$ is 


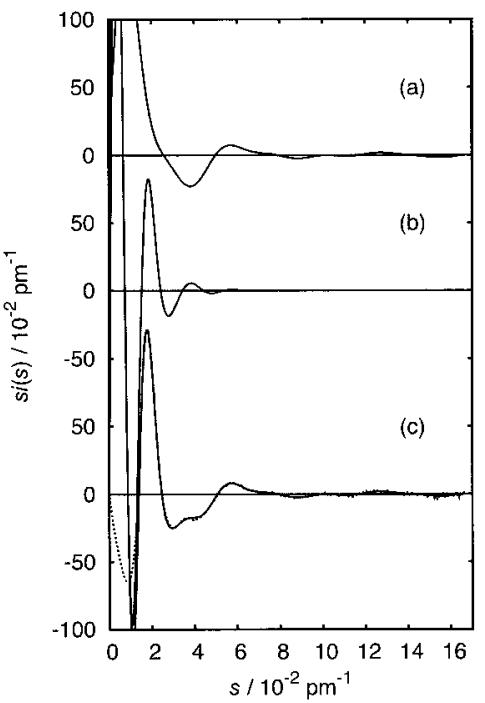

Fig. 2 The si $(s)$ curve for acetonitrile. The calculated values for the intramolecular and intermolecular interactions are shown in (a) and (b), respectively. The dotted and solid lines in (c) represent the observed and calculated curves, respectively.

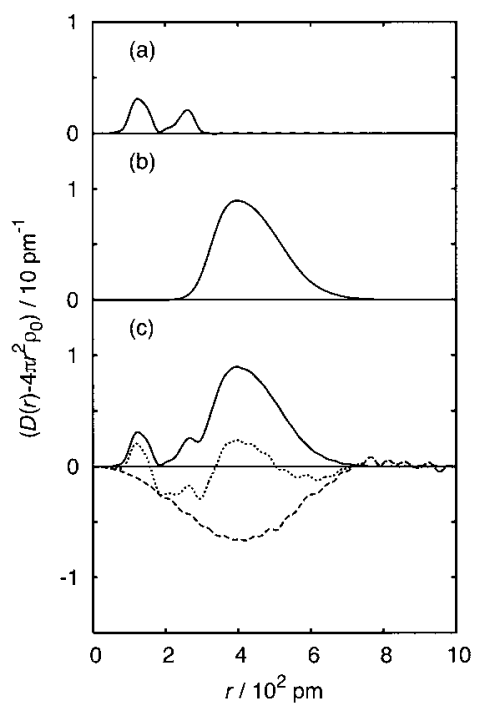

Fig. 3 The $\left(D(r)-4 \pi r^{2} \rho_{0}\right)$ curve for acetonitrile. The calculated peak shapes for the intramolecular and intermolecular interactions are shown in (a) and (b), respectively. (c) The dashed line indicates the residual curve after subtracting the calculated curve (solid line) from the experimentally obtained one (dotted line).

depicted in Fig. 3. In the curve the peak at $130 \mathrm{pm}$ is ascribed to the $\mathrm{C}-\mathrm{H}, \mathrm{C}-\mathrm{C}$ and $\mathrm{C} \equiv \mathrm{N}$ bonds. The intramolecular nonbonding $\mathrm{C} \cdots \mathrm{H}$ and $\mathrm{C} \cdots \mathrm{N}$ pairs contribute to the peak at 260 $\mathrm{pm}$ with a hump at $220 \mathrm{pm}$. The structure parameters of these interactions were refined by a least-squares procedure applied to the $s i(s)$ curve in the region of $5<s / 10^{-2} \mathrm{pm}^{-1}<16$. The electron diffraction results of gaseous acetonitrile were referred to the $\mathrm{C}-\mathrm{H}, \mathrm{C}-\mathrm{C}$, and $\mathrm{C} \equiv \mathrm{N}$ bond distances ${ }^{33}$ and these values were fixed in the course of the optimization. The results are listed in Table 1; almost the same results were obtained by using a different fitting region of $6<s / 10^{-2} \mathrm{pm}^{-1}<16$.

Since the intermolecular interactions appear in a longer region than the intramolecular interactions, the $s i(s)$ data in a small $s$ region of $1<s / 10^{-2} \mathrm{pm}^{-1}<5$ was used for the least-squares
Table 1 Intramolecular structure parameters for liquid acetonitrile $^{\mathrm{a}}$

\begin{tabular}{lccc}
\hline Interaction & $r / \mathrm{pm}$ & $b / 10 \mathrm{pm}^{2}$ & $n$ \\
\hline $\mathrm{C}-\mathrm{H}$ & $111^{\mathrm{b}}$ & $1^{\mathrm{b}}$ & $3^{\mathrm{b}}$ \\
$\mathrm{C} \equiv \mathrm{N}$ & $116^{\mathrm{b}}$ & $1^{\mathrm{b}}$ & $1^{\mathrm{b}}$ \\
$\mathrm{C}\left(\mathrm{CH}_{3}\right)-\mathrm{C}$ & $147^{\mathrm{b}}$ & $1^{\mathrm{b}}$ & $1^{\mathrm{b}}$ \\
$\mathrm{C} \cdots \mathrm{H}$ & $223(7)$ & $1^{\mathrm{b}}$ & $3^{\mathrm{b}}$ \\
$\mathrm{C}\left(\mathrm{CH}_{3}\right) \cdots \mathrm{N}$ & $262(1)$ & $6(1)$ & $1^{\mathrm{b}}$ \\
\hline
\end{tabular}

a. Standard deviations are given in parentheses.

b. The values were kept constant during the calculation.

Table 2 Intermolecular structure parameters for liquid acetonitrile ${ }^{\mathrm{a}, \mathrm{b}}$

\begin{tabular}{crrrcrrr}
\hline Molecule & \multicolumn{1}{c}{$\psi /^{\circ}$} & \multicolumn{1}{c}{$\theta /^{\circ}$} & \multicolumn{1}{c}{$\phi /^{\circ}$} & $R / \mathrm{pm}$ & \multicolumn{1}{c}{$\Theta /^{\circ}$} & \multicolumn{1}{c}{$\Phi /^{\circ}$} & $B$ \\
\hline A & $157(7)$ & $2(4)$ & $17(6)$ & $212(21)$ & $27(5)$ & $17(4)$ & $0.1^{\mathrm{c}}$ \\
$\mathrm{B}$ & $3(5)$ & $14(5)$ & $2(5)$ & $227(27)$ & $-86(4)$ & $-63(4)$ & $0.1^{\mathrm{c}}$ \\
$\mathrm{C}$ & $-16(12)$ & $6(4)$ & $6(11)$ & $380(16)$ & $83(3)$ & $-30(3)$ & $0.1^{\mathrm{c}}$
\end{tabular}

a. The values in parentheses are standard deviations.

b. Cartesian coordinates of atoms with no atomic scattering power in an acetonitrile molecule situated at the origin are as follows.

\begin{tabular}{crcc}
\hline Atom & $x / 10^{2} \mathrm{pm}$ & $y / 10^{2} \mathrm{pm}$ & $z / 10^{2} \mathrm{pm}$ \\
\hline $\mathrm{N}$ & 1.441 & 0 & -0.001 \\
$\mathrm{C}$ & 0.281 & 0 & -0.001 \\
$\mathrm{C}\left(\mathrm{CH}_{3}\right)$ & -1.189 & 0 & -0.001 \\
\hline
\end{tabular}

c. The values were kept constant during the calculation.

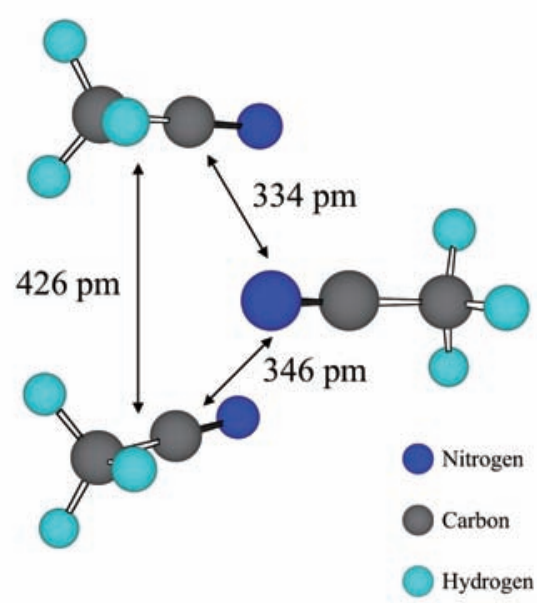

Fig. 4 Liquid structure of acetonitrile.

fitting to obtain the intermolecular structural parameters of liquid acetonitrile. Three acetonitrile molecules are enough to reproduce the X-ray scattering data well. The intermolecular structural parameters obtained are listed in Table 2. If the fitting region was varied, $1<s / 10^{-2} \mathrm{pm}^{-1}<4$ or $1<s / 10^{-2} \mathrm{pm}^{-1}<6$, the results in Table 2 are practically unchanged. The molecular arrangement calculated from the parameters is depicted in Fig. 4. The picture for the liquid acetonitrile clearly shows the zigzag arrangement, which is the same as that reported in the past studies. ${ }^{15-19}$ The average $\mathrm{C} \cdots \mathrm{N}$ distance between the nearest neighbor molecules is $340 \mathrm{pm}$, which agreed well with the literature values $\left(330^{17}, 335^{15}, 340^{19}\right.$, and $\left.345^{18} \mathrm{pm}\right)$. The $\mathrm{N} \cdots \mathrm{N}$, 


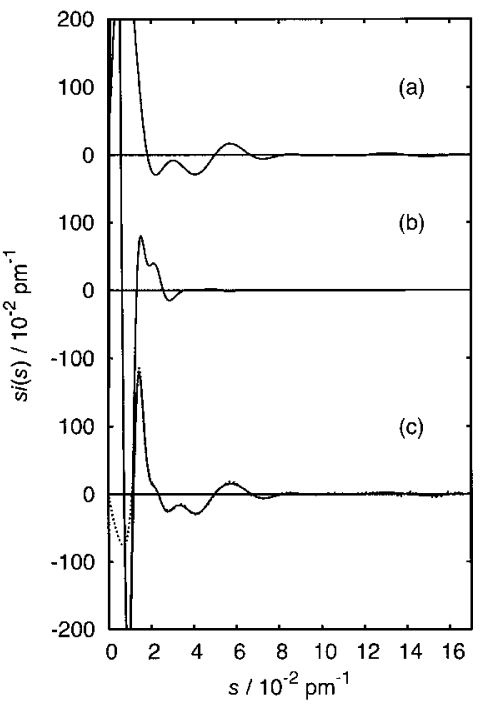

Fig. 5 The si $(s)$ curve for acetone. The calculated values for the intramolecular and intermolecular interactions are shown in (a) and (b), respectively. The dotted and solid lines in (c) represent the observed and calculated curves, respectively.

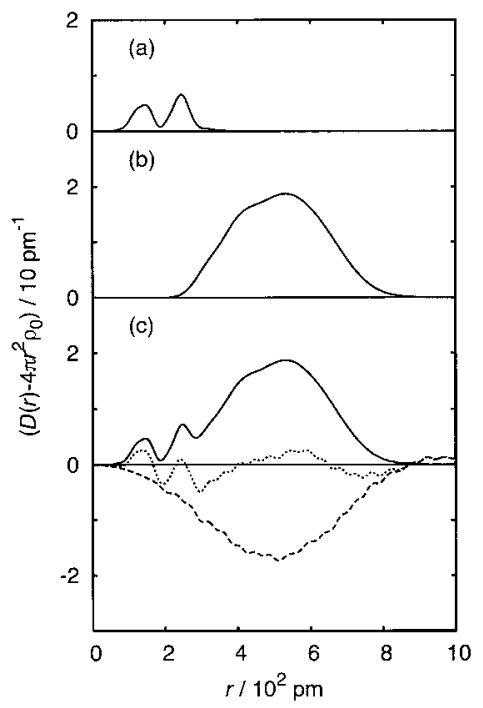

Fig. 6 The $\left(D(r)-4 \pi r^{2} \rho_{0}\right)$ curve for acetone. The calculated peak shapes for the intramolecular and intermolecular interactions are shown in (a) and (b), respectively. (c) The dashed line indicates the residual curve after subtracting the calculated curve (solid line) from the experimentally obtained one (dotted line).

C $\cdots \mathrm{C}$ and $\mathrm{C}\left(\mathrm{CH}_{3}\right) \cdots \mathrm{C}\left(\mathrm{CH}_{3}\right)$ distances between the second nearest molecules are 395, 423 and $460 \mathrm{pm}$, respectively.

\section{Liquid Acetone}

The si(s) and $\left(D(r)-4 \pi r^{2} \rho_{0}\right)$ curves for liquid acetone are depicted in Figs. 5 and 6, respectively. The peak at $140 \mathrm{pm}$ in the $\left(D(r)-4 \pi r^{2} \rho_{0}\right)$ curve is due to the $\mathrm{C}-\mathrm{H}, \mathrm{C}-\mathrm{C}$ and $\mathrm{C}-\mathrm{O}$ bonds. The peak at $240 \mathrm{pm}$ is ascribed to the intramolecular nonbonding $\mathrm{C} \cdots \mathrm{O}, \mathrm{C}\left(\mathrm{CH}_{3}\right) \cdots \mathrm{C}\left(\mathrm{CH}_{3}\right)$ and $\mathrm{C} \cdots \mathrm{H}$ pairs. The structure parameters for the intramolecular interactions were refined by the least-squares method in the $s$ space and the fitting
Table 3 Intramolecular structure parameters for liquid acetone ${ }^{\mathrm{a}}$

\begin{tabular}{lccc}
\hline \multicolumn{1}{c}{ Interaction } & $r / \mathrm{pm}$ & $b / 10 \mathrm{pm}^{2}$ & $n$ \\
\hline $\mathrm{C}-\mathrm{H}$ & $110^{\mathrm{b}}$ & $1^{\mathrm{b}}$ & $6^{\mathrm{b}}$ \\
$\mathrm{C}=\mathrm{O}$ & $121^{\mathrm{b}}$ & $1^{\mathrm{b}}$ & $1^{\mathrm{b}}$ \\
$\mathrm{C}\left(\mathrm{CH}_{3}\right)-\mathrm{C}$ & $152^{\mathrm{b}}$ & $1^{\mathrm{b}}$ & $2^{\mathrm{b}}$ \\
$\mathrm{C} \cdots \mathrm{H}$ & $215^{\mathrm{c}}$ & $2^{\mathrm{b}}$ & $6^{\mathrm{b}}$ \\
$\mathrm{C}\left(\mathrm{CH}_{3}\right) \cdots \mathrm{O}$ & $241(1)$ & $10(4)$ & $2^{\mathrm{b}}$ \\
$\mathrm{C}\left(\mathrm{CH}_{3}\right) \cdots \mathrm{C}\left(\mathrm{CH}_{3}\right)$ & $255(2)$ & $6(5)$ & $1^{\mathrm{b}}$ \\
\hline
\end{tabular}

a. Standard deviations are given in parentheses.

b. The values were kept constant during the calculation.

c. Assumed that the $\mathrm{H}-\mathrm{C}-\mathrm{C}$ bond angle is $109.5^{\circ}$.

Table 4 Intermolecular structure parameters for liquid acetone $^{\mathrm{a}, \mathrm{b}}$

\begin{tabular}{crrrrrrr}
\hline Molecule & \multicolumn{1}{c}{$\psi /^{\circ}$} & \multicolumn{1}{c}{$\theta /^{\circ}$} & \multicolumn{1}{c}{$\phi /^{\circ}$} & \multicolumn{1}{c}{$R / \mathrm{pm}$} & \multicolumn{1}{c}{$\Theta /^{\circ}$} & \multicolumn{1}{c}{$\Phi /^{\circ}$} & $B$ \\
\hline A & $21(2)$ & $33(1)$ & $-3(2)$ & $337(13)$ & $-75(3)$ & $-96(2)$ & $0.1^{\mathrm{c}}$ \\
$\mathrm{B}$ & $219(2)$ & $27(2)$ & $16(2)$ & $362(5)$ & $56(2)$ & $-8(2)$ & $0.1^{\mathrm{c}}$ \\
$\mathrm{C}$ & $216(2)$ & $15(1)$ & $185(2)$ & $223(13)$ & $270(3)$ & $97(2)$ & $0.1^{\mathrm{c}}$
\end{tabular}

a. The values in parentheses are standard deviations.

b. Cartesian coordinates of atoms with no atomic scattering power in an acetone molecule situated at the origin are as follows.

\begin{tabular}{cccc}
\hline Atom & $x / 10^{2} \mathrm{pm}$ & $y / 10^{2} \mathrm{pm}$ & $z / 10^{2} \mathrm{pm}$ \\
\hline $\mathrm{O}$ & 1.395 & 0 & 0 \\
$\mathrm{C}$ & 0.185 & 0 & 0 \\
$\mathrm{C}\left(\mathrm{CH}_{3}\right)$ & -0.613 & 1.290 & 0 \\
$\mathrm{C}\left(\mathrm{CH}_{3}\right)$ & -0.613 & -1.290 & 0 \\
\hline
\end{tabular}

c. The values were kept constant during the calculation.

region was $4<s / 10^{-2} \mathrm{pm}^{-1}<16,5<s / 10^{-2} \mathrm{pm}^{-1}<16$, or $6<s / 10^{-2} \mathrm{pm}^{-1}<16$. The $\mathrm{C}-\mathrm{H}, \mathrm{C}-\mathrm{C}$ and $\mathrm{C}-\mathrm{O}$ bond distances were taken from the literature. ${ }^{34}$ The intramolecular structure parameters optimized are essentially the same independent of the $s$ range. The results obtained by using $5<s / 10^{-2} \mathrm{pm}^{-1}<16$ are summarized in Table 3 .

The intermolecular structural parameters of liquid acetone were obtained by the least-squares fitting applied to the $s i(s)$ data in a region of $1<s / 10^{-2} \mathrm{pm}^{-1}<5$. In contrast to the result by Bertagnolli et al.,22 the X-ray scattering data were well reproduced in terms of a cluster consisting of three acetone molecules. The structural parameters for the intermolecular interactions are listed in Table 4. The residual curve (the dashed line in Fig. 6) after subtracting the peaks due to both intramolecular and intermolecular interactions is smooth, with no distinct peak over the range $r<1000 \mathrm{pm}$. As seen in Fig. 5, the $s i(s)$ curve arising from the intermolecular interactions displays no oscillation at $s>c a .3 .5$ and the change of $s_{\max }$ value (4 or 6) in Eq. (10) did not affect the results. Figure 7 shows the liquid structure of acetone and one sees that the molecular arrangement is similar to that found in the crystal. In the stable phase of solid acetone at ambient pressure and $150 \mathrm{~K}$, there exist two kinds of intermolecular $\mathrm{C} \cdots \mathrm{O}$ distances. ${ }^{35}$ One distance results from two acetone molecules interacting with their $-\mathrm{C}=\mathrm{O}$ groups in an antiparallel fashion and is $330.0 \mathrm{pm}$. The other is the distance between two acetone molecules orienting perpendicularly and is $345.8 \mathrm{pm}$. Thus, the dipoledipole interaction in an antiparallel way is strong in solid phase. As seen in Fig. 7, the average antiparallel C... O distance is 379 pm while the perpendicular one is $306 \mathrm{pm}$ for liquid acetone. 


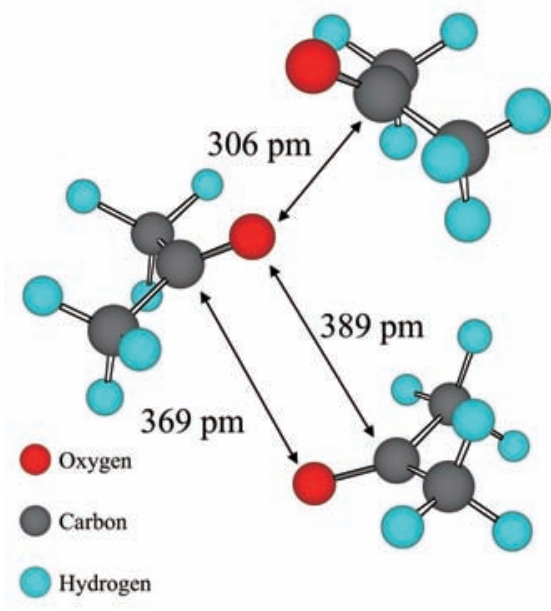

Fig. 7 Liquid structure of acetone.

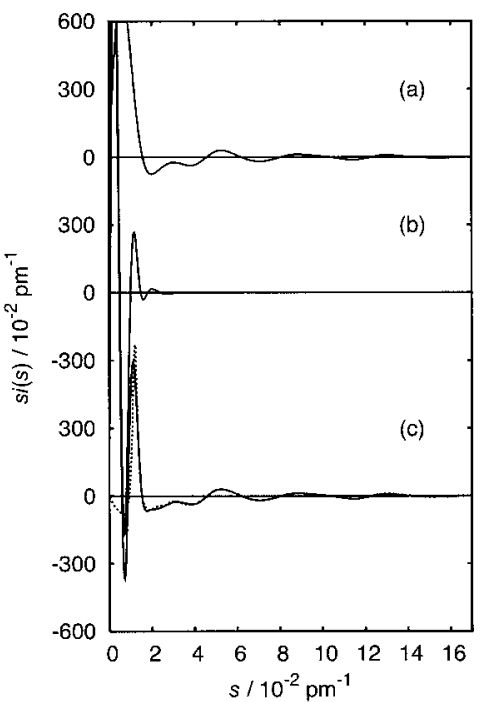

Fig. 8 The si(s) curve for cyclohexane. The calculated values for the intramolecular and intermolecular interactions are shown in (a) and (b), respectively. The dotted and solid lines in (c) represent the observed and calculated curves, respectively.

Therefore, the perpendicular interaction is predominant in a liquid state.

\section{Liquid Cyclohexane}

Figures 8 and 9 show the si(s) and $\left(D(r)-4 \pi r^{2} \rho_{0}\right)$ curves for cyclohexane, respectively. The peak at $140 \mathrm{pm}$ in the $(D(r)-$ $\left.4 \pi r^{2} \rho_{0}\right)$ curve is attributable to the $\mathrm{C}-\mathrm{H}$ and $\mathrm{C}-\mathrm{C}$ bonds. The broad peak centered at $260 \mathrm{pm}$ consists of the peaks due to the intramolecular nonbonding $\mathrm{C} \cdots \mathrm{C}$ and $\mathrm{C} \cdots \mathrm{H}$ pairs. The hump around $380 \mathrm{pm}$ is ascribable to the nonbonding $\mathrm{C} \cdots \mathrm{H}$ pair. Since the number of the pair is 24 , this interaction must not be neglected in the analyses. Two conformers, chair and boat, are possible for a cyclohexane molecule. Farman et al. concluded that both chair and boat conformers are comparably present; the boat conformation is slightly preferable based on their neutron scattering data. ${ }^{23}$ However, the vibrational spectroscopic and thermodynamic data were interpreted in terms of the predominant

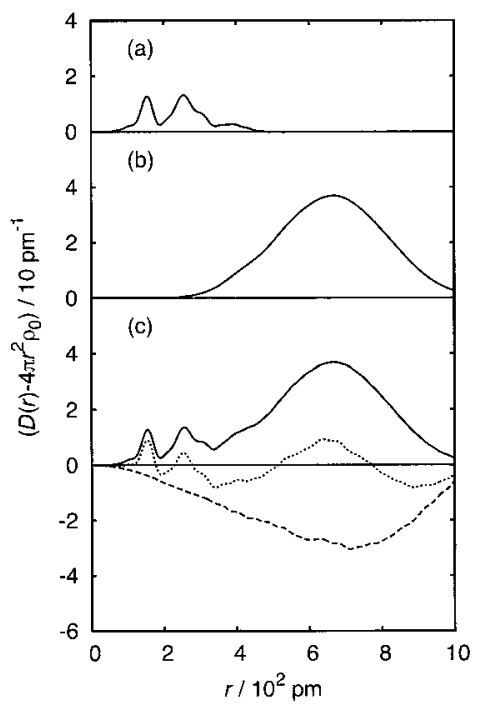

Fig. 9 The $\left(D(r)-4 \pi r^{2} \rho_{0}\right)$ curve for cyclohexane. The calculated peak shapes for the intramolecular and intermolecular interactions are shown in (a) and (b), respectively. (c) The dashed line indicates the residual curve after subtracting the calculated curve (solid line) from the experimentally obtained one (dotted line).

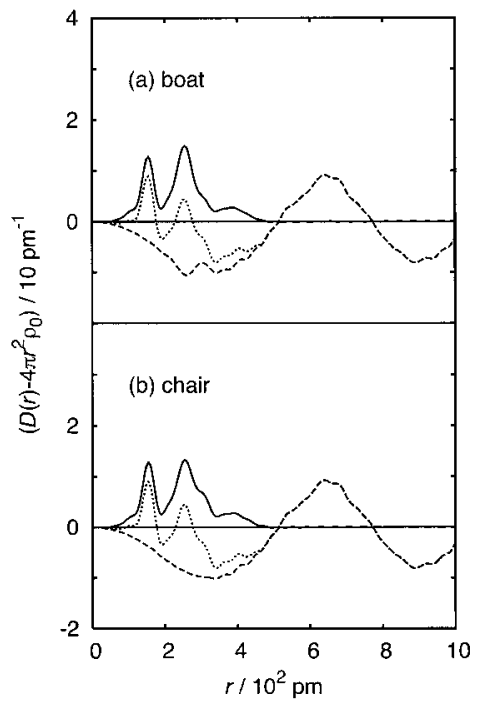

Fig. 10 Comparison of the calculated peak shapes (solid lines) for the boat and chair conformers of cyclohexane. The dotted lines stand for the experimental $\left(D(r)-4 \pi r^{2} \rho_{0}\right)$ curve and the dashed lines are the residual curves.

existence of the chair conformer. ${ }^{36}$ Moreover, temperaturedependent NMR spectra are only devoted to the determination of the kinetic parameters for the chair $\leftrightarrow$ chair interconversion. ${ }^{37-40}$ There are two kinds of the diagonal $\mathrm{C} \cdots \mathrm{C}$ distances in a cyclohexane molecule. The shorter C ... C distance appears at $255 \mathrm{pm}$ commonly for the chair and boat conformers. The longer distance for three C $\cdots \mathrm{C}$ pairs is $300 \mathrm{pm}$ for the chair conformer while the same distance for two C … C pairs and a distance of $275 \mathrm{pm}$ for one $\mathrm{C} \cdots \mathrm{C}$ pair exist for the boat conformation. Figure 10 compares the calculated peak shapes due to the chair and boat forms which are drawn by assuming that the respective conformers are present in the neat liquid at the rate of $100 \%$. The intramolecular structure parameters for the chair conformer 
Table 5 Intramolecular structure parameters for liquid cyclohexane $^{\mathrm{a}}$

\begin{tabular}{cccr}
\hline Interaction & $r / \mathrm{pm}$ & $b / 10 \mathrm{pm}^{2}$ & $n$ \\
\hline $\mathrm{C}-\mathrm{H}$ & $112^{\mathrm{b}}$ & $1^{\mathrm{b}}$ & $12^{\mathrm{b}}$ \\
$\mathrm{C}-\mathrm{C}$ & $154^{\mathrm{b}}$ & $1^{\mathrm{b}}$ & $6^{\mathrm{b}}$ \\
$\mathrm{C} \cdots \mathrm{H}$ & $218^{\mathrm{c}}$ & $2^{\mathrm{b}}$ & $24^{\mathrm{b}}$ \\
$\mathrm{C} \cdots \mathrm{C}$ & $256(1)$ & $8(1)$ & $6^{\mathrm{b}}$ \\
$\mathrm{C} \cdots \mathrm{H}$ & $265(5)$ & $8^{\mathrm{b}}$ & $12^{\mathrm{b}}$ \\
$\mathrm{C} \cdots \mathrm{C}$ & $303(1)$ & $6(1)$ & $3^{\mathrm{b}}$ \\
$\mathrm{C} \cdots \mathrm{H}$ & $375(5)$ & $40^{\mathrm{b}}$ & $24^{\mathrm{b}}$ \\
\hline
\end{tabular}

a. Standard deviations are given in parentheses.

b. The values were kept constant during the calculation.

c. Assumed that the $\mathrm{H}-\mathrm{C}-\mathrm{C}$ bond angle is $109.5^{\circ}$.

Table 6 Intermolecular structure parameters for liquid cyclohexane ${ }^{\mathrm{a}, \mathrm{b}}$

\begin{tabular}{crrrcrrr}
\hline Molecule & $\psi$ & \multicolumn{1}{c}{$\theta /^{\circ}$} & \multicolumn{1}{c}{$\phi l^{\circ}$} & $R / \mathrm{pm}$ & $\Theta /^{\circ}$ & \multicolumn{1}{c}{$\Phi /^{\circ}$} & $B$ \\
\hline A & $-16(3)$ & $-159(4)$ & $-84(4)$ & $409(8)$ & $-60(3)$ & $51(2)$ & $0.1^{\mathrm{c}}$ \\
$\mathrm{B}$ & $-73(2)$ & $232(2)$ & $96(2)$ & $291(9)$ & $126(3)$ & $13(2)$ & $0.1^{\mathrm{c}}$ \\
$\mathrm{C}$ & $61(2)$ & $33(2)$ & $-33(2)$ & $396(9)$ & $238(2)$ & $-53(2)$ & $0.1^{\mathrm{c}}$ \\
\hline
\end{tabular}

a. The values in parentheses are standard deviations.

b. Cartesian coordinates of atoms with no atomic scattering power in a cyclohexane molecule situated at the origin are as follows.

\begin{tabular}{cccc}
\hline Atom & $x / 10^{2} \mathrm{pm}$ & $y / 10^{2} \mathrm{pm}$ & $z / 10^{2} \mathrm{pm}$ \\
\hline $\mathrm{C}$ & 1.381 & 0 & -0.576 \\
$\mathrm{C}$ & 0.768 & 1.285 & 0 \\
$\mathrm{C}$ & -0.768 & 1.285 & 0 \\
$\mathrm{C}$ & -1.381 & 0 & 0.576 \\
$\mathrm{C}$ & -0.768 & -1.285 & 0 \\
$\mathrm{C}$ & 0.768 & -1.285 & 0 \\
\hline
\end{tabular}

c. The values were kept constant during the calculation.

were quoted from the literature. ${ }^{41}$ The intramolecular distances for the boat conformer were estimated by moving one $-\mathrm{CH}_{2-}$ group of the chair conformer so that the inversion center disappears in the chair conformer and a new plane of symmetry appears in the boat conformer. It is obvious that the chair conformer leads to the smooth background curve but the boat conformer does not. The structure parameters for the intramolecular interactions were determined in the $s$ space by the least-squares method in the range of $3<s / 10^{-2} \mathrm{pm}^{-1}<16$ or $4<s / 10^{-2} \mathrm{pm}^{-1}<16$. Regardless of the $s$ regions, similar results were obtained. Such results are listed in Table 5. Our least-squares calculations revealed that the distance of $275 \mathrm{pm}$ initially inserted for one C...C pair approached to a value close to $300 \mathrm{pm}$ and the presence of the boat conformer was rejected. We concluded that the chair conformer predominantly exists in the liquid phase.

The intermolecular interactions in liquid cyclohexane were optimized at the fixed conformation of the chair form by using the $s i(s)$ data in a range of $1<s / 10^{-2} \mathrm{pm}^{-1}<5$. The effect of the fitting region on the final results were checked by a similar procedure to that employed for acetone. The peak over the region of $500-800 \mathrm{pm}$ in the $\left(D(r)-4 \pi r^{2} \rho_{0}\right)$ curve is from the nearest neighbor interactions. This peak was well explained by considering three cyclohexane molecules. The structural parameters for the intermolecular interactions are listed in Table 6. The liquid structure of cyclohexane is shown in Fig. 11. The distances between the centers of three molecules are 578, 596,

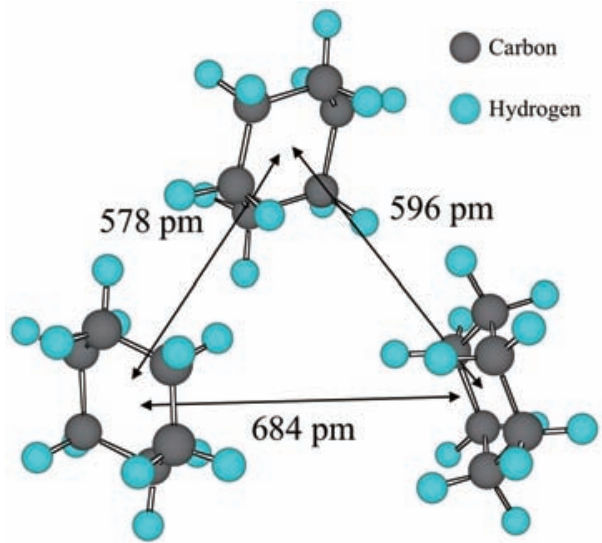

Fig. 11 Liquid structure of cyclohexane.

and $684 \mathrm{pm}$. A glance at Fig. 11 tells us that no specific interaction operates and that the liquid structure is disordered. In such a case, the determination of liquid structure by the present procedure is especially useful.

\section{Acknowledgements}

This work was supported in part by "Academic Frontier" Project for Private Universities: matching fund subsidy from the Ministry of Education, Culture, Sports, Science and Technology of Japan, 2003 - 2007.

\section{References}

1. P. Debye and P. Scherrer, Nachr. Ges. Wiss. Göttingen, 1916, 1,1 .

2. H. Ohtaki, T. Radnai, and T. Yamaguchi, Chem. Soc. Rev., 1997, 41.

3. M. Nakahara, T. Yamaguchi, and H. Ohtaki, Recent Res. Devel. Phys. Chem., 1997, 1, 17.

4. G. W. Stewart, Phys. Rev., 1929, 33, 889.

5. S. Katzoff, J. Chem. Phys., 1934, 2, 841.

6. R. H. Kruh, Chem. Rev., 1962, 62, 319.

7. H. Ohtaki and K. Ozutsumi, Russ. J. Phys. Chem., 2003, 77, S66.

8. A. H. Narten, ORNL-4578, 1970.

9. A. H. Narten, J. Chem. Phys., 1968, 48, 1630.

10. H. Ohtaki and S. Itoh, Z. Naturforsch., 1985, 40a, 1351.

11. S. Itoh and H. Ohtaki, Z. Naturforsch., 1987, 42a, 858.

12. S. Trabelsi and S. Nasr, J. Chem. Phys., 2004, 121, 6380.

13. T. Iijima and K. Nishikawa, Chem. Phys. Lett., 1985, 115, 522.

14. K. Nishikawa and T. Iijima, Bull. Chem. Soc. Jpn., 1986, 59, 117.

15. V. A. Kratochwill, J. U. Weidner, and H. Zimmermann, Ber. Bunsenges. Phys. Chem., 1973, 77, 408.

16. H. Bertagnolli and M. D. Zeidler, J. Mol. Liq., 1978, 35, 177.

17. T. Radnai, S. Itoh, and H. Ohtaki, Bull. Chem. Soc. Jpn., 1988, 61, 3845.

18. T. Takamuku, M. Tabata, A. Yamaguchi, J. Nishimoto, M. Kumamoto, H. Wakita, and T. Yamaguchi, J. Phys. Chem. $B, \mathbf{1 9 9 8}, 102,8880$.

19. M. Katayama, K. Komori, K. Ozutsumi, and H. Ohtaki, Z. Phys. Chem., 2004, 218, 659. 
20. R. L. McGreevy and L. Pusztai, Proc. R. Soc. Lond. A, 1990, 430, 241.

21. A. K. Soper, J. Mol. Liq., 1998, 78, 179.

22. H. Bertagnolli, M. Hoffmann, and M. Ostheimer, Z. Phys. Chem. (Neue Folge), 1989, 165, 165.

23. H. Farman, J. C. Dore, M.-C. Bellisent-Funel, and D. G. Montague, Mol. Phys., 1987, 61, 583.

24. Z. Bochyński and H. Drozdowski, Acta Phys. Slovaca, 1999, 49, 409.

25. L. Azaroff, Acta Crystallogr., 1955, 8, 701.

26. B. Warren and R. Mozzi, Acta Crystallogr., 1966, 21, 459.

27. J. Krough-Moe, Acta Crystallogr., 1956, 9, 951.

28. N. Norman, Acta Crystallogr., 1957, 10, 370.

29. D. T. Cromer, J. Chem. Phys., 1969, 50, 4857.

30. D. T. Cromer and J. Liberman, J. Chem. Phys., 1970, 53, 1891.

31. G. Johansson and M. Sandström, Chem. Scripta, 1973, 4, 195.
32. D. W. Marquardt, J. Soc. Ind. Appl. Math., 1963, 11, 431.

33. K. Karakida, T. Fukuyama, and K. Kuchitsu, Bull. Chem. Soc. Jpn., 1974, 47, 299.

34. T. Iijima, Bull. Chem. Soc. Jpn., 1972, 45, 3526.

35. D. R. Allan, S. J. Clark, R. M. Ibberson, S. Parsons, C. R. Pulham, and L. Sawyer, Chem. Commun., 1999, 751.

36. C. W. Beckett, K. Pitzer, and R. Spitzer, J. Am. Chem. Soc., 1947, 69, 2488.

37. F. R. Jensen, D. S. Noyce, C. H. Sederholm, and A. J. Berrlin, J. Am. Chem. Soc., 1960, 82, 1256.

38. A. Allerhand, F.-M. Chen, and H. S. Gutowsky, J. Chem. Phys., 1965, 42, 3040.

39. F. A. L. Anet and A. J. R. Bourn, J. Am. Chem. Soc., 1967, 89,760

40. R. Poupko and Z. Luz, J. Chem. Phys., 1981, 75, 1675.

41. O. Bastiansen, L. Fernholt, H. M. Seip, H. Kambara, and K. Kuchitsu, J. Mol. Struct., 1973, 18, 163. 